\title{
VARIABILIDAD MORFOLOGICA DE LERNAEA CYPRINACEA (CRUSTACEA: COPEPODA) EN EL EMBALSE DE ARROCAMPO (CUENCA DEL TAJO: CACERES)
}

\author{
O. Moreno, C. Granado, F. García Novo \\ Dpto.Ecología.Facultad de Biología. Universidad de Sevilla.
}

Palabras clave: Lernaea cyprinacea, morphology, insertion sites.

\author{
ABSTRACT \\ MORPHOLOGICAL VARIABILITY OF LERNAEA CYPRINACEA (CRUSTACEA: COPEPODA) \\ IN ARROCAMPO RESERVOIR TAJO BASIN: CACERES
}

\begin{abstract}
The most relevant modifications in the morphology of cephalic area were studied in Lernaea cyprinacea. Thirteen morphologycal types of anchor arms were found, this character being unrelated to the number of pregenital prominences. Insertion sites on the host determine morphological features of anchor arms. The variability of forms is found to be higher in the gills and body surface, and lower in fins. This fact causes the aparition of specific morphological types in these areas.
\end{abstract}

\section{INTRODUCCION}

Los hábitos de vida parásitos llevan consigo, en muchos casos, la simplificación de la morfología externa de la especie y una evolución notable de los órganos de sujeción al hospedador.

La morfología de Lernaea cyprinacea, copepodo parásito de peces de agua dulce, llevó a LINNEO, quién la describió en 1758, a incluirla dentro de los «vermes Moluscos». Unicamente la presencia de sacos ovígeros y trazas de segmentación en el abdómen, muy alargado, indica que esta especie pertenece a los Crustáceos Copepodos. (Figura 1).

La descripción en 1925 de Lernaea elegans por LEIGH-SHARPE sobre Anguilla japonica, originó en años posteriores controversia sobre la identificación de la especie que se presentaba en Europa. Nakai (en Harding, 1950) apoya a Leing-Sharpe (1925) en considerar la especie que aparece en Extremo Oriente como L. Elegans, mientras que Hu (en Fryer, 1961) considera un complejo de formas dándole valor subespecífico, tanto a $L$. elegans como a $L$. cyprinacea (formas oriental y occidental respectivamente), basándose en caracteres morfológicos (número de artejos y setas de !os apéndices o distancia de los segmentos abdominales con respecto a la longitud total).

Limnetica 2 265-270(1986)

(C) Asociación Española de Limnología, Madrid Spain
Utilizando la morfología de la región cefálica y el número de prominencias pregenitales como caracteres para diferenciar especies dentro del género Lernaea, Harding (1950) aprecia que es posible distinguir dentro de $L$. cyprinacea una forma oriental con el brazo dorsal en forma de $\mathrm{Y}$ y una forma occidental en $\mathrm{T}$. siendo estos los caracteres utilizados anteriormente para distinguir ambas especies (L. elegans en forma de Y y L. cyprinacea en forma de T). Este autor apunta además que los caracteres utilizados por Leigh-Sharpe (1925), en su descripción no sirven para distinguir una especie de otra, y que otras características citadas para esta especie, como las «expansiones auriculares» no se observan en el ejemplar tipo de la misma.

Otros autores (Fryer, 1961; Fratello et al., 1972; Amin, 1981; Eisen, 1981) consideran que las variaciones morfológicas en el brazo dorsal se deben a causas ambientales (sitio de unión al hospedador, temperatura del agua, etc.) y que ambas especies son sinónimas, ya que las variaciones morfológicas son tan amplias en cada estudio que no es posible distinguir los ejemplares occidentales de la descripción de la forma oriental.

Otro grupo de autores, sin embargo, apoyan la distinción de dos especies en este grupo de Lernaea: Hoffmann (1976) considera las diferencias morfológi- 
Tipos

cefálicos
I
II
II
V
VI
VII
VIII
IX
X
XI
XII
XIII

Total
N. ${ }^{\circ}$ de prominencias pregenitales

$\begin{array}{rrrr}\% & \text { Dos } & \% & \text { Total } \\ 78 & 4 & 22 & 18 \\ 75 & 4 & 25 & 16 \\ 71 & 5 & 29 & 17 \\ 100 & 0 & 0 & 2 \\ 67 & 1 & 33 & 3 \\ 0 & 1 & 100 & 1 \\ 50 & 1 & 50 & 2 \\ 100 & 0 & 0 & 1 \\ 75 & 1 & 25 & 4 \\ 100 & 0 & 0 & 1 \\ 0 & 1 & 100 & 1 \\ 100 & 0 & 0 & 1 \\ 100 & 0 & 0 & 68\end{array}$

Tabla 1.-Frecuenciade individuos con una o dos prominencias pregenitales en función del tipo de región cefálica en $L$. cyprrnac'éa Frequency of individuals with one or two pregenital prominences as a function of the type ofanchor in $L$. cyprinacea.

cas del brazo dorsal suficientes para distinguir dos especies. Poddubnaya (1974) restringe L. cyprinacea a Carassius carassiuts considerandu a L. clegans como parásito con menor especificidad por el hospedador.

Por el contrario, Frycr $(1956,1961,1982)$, Amin (1973, 1981). Fratello et al. (1972), Eisen (1981) han encontrado a $L$. cyprinacea, considerada según los criterios de Harding (op. cit.), en distintos hospedadores de familias muy diversas, que permiten catalogar a este parásito conio no especifico.

La utilización de otros caracteres como las prominencias pregenitalcs también ha suscitado controversia. Harding (op. cit.) lo utilizó para diferenciar especies, mientras que Fryer (1961) no le concede valor taxonómico y Eisen (op. cit.). además observa que depende de la especie de hospedador.

\section{MATERIAL Y METODOS}

En el presente trabajo se han estudiado la variabilidad morfológica de Le'mata clprinacé en función del lugar de inserción sobre el pez, en un estudio icliológico llevado a cabo en el embalse de Arrocampo (afluente del rio Tajo).

Los ejemplares examinados, presentes en la boga de rio (chondrostoma polylepis polylepis, fueron extraidos de la epidermis del pez, tipificándose la región donde se localizaban. Se distinguieron 36 zonas repartidas en: cabeza (una zona. 1): branquias (una zona. 2): aletas (seis zonas. 21. 22. 33. 24, 25. 26): s superficie del cuerpo (dieciocho zonas. 3. ..., 30) distribuyéndose estas últimas en sentido longitudinal y transversal a lo largo de todo el pez (figura 3 ).

Se realizó un dibujo esquemático de la región cefálica y se determinó el número de prominericius pregenitales que presentaba cada ejemplar.

\section{RESULIADOS}

En los 94 cjemplares examinados se distinguieron 13 tipos morfológicos de la región cefálica (figura 3 ). basadas en las variaciones de inclinación y abertura de las ramas y orientación de los brazos.

El porcentaje de aparición de cada tipo cefálico varía entre 1.06 y $23.4 \%$. Aquellos que se presentan con mayor proporción son los tipos 3, 1, 2 y 10 con 23.4 . $21.3,14.9$ y $12.8 \%$ respectivamente. Los tres primeros con los brazos en forma de $\mathrm{T}$ y el último en forma dé Y. En relación a este criterio, se observó un 72.4\% de los ejemplares en I (tipos 1 a 8 ) y un 27.6\% (tipos 9 a 13 ) en Y.

Los tipos 1 y 2 presentan la forma típica descrita para t. crprmaced (Harding, 1950; Fryer, 1961. 1982: (irabda. 1903). El tipo 0 corresponde igualmente al considerado, según Hofmann (op. cit.). para 1. ckegans, nientras que los demás tipos suponen variaciones con respecto a estas dos formas

Para ver su consistencia como carácter taxonómico a utilizar. se relaciono ha hecuencia de aparicion del numero de prominencias pregenitales con la morfolo- 
Total

65

94

Tipos

cefalicos

1

II

III

IV

V

VI

VII

VIII

IX

$\mathrm{X}$

$\mathrm{XI}$
$\times \mathrm{II}$

XIII

Total

4

N
H

0.70

0.58

0.30

0.41

0.46

0.60

0.41

0.64

0.48

0.45

0. 30

0.41

$0+1$

0.76

0.30

0.30

0.56

$H$

1.21

0.83

0.99

0.47

1.00

0.30

0.82

0.67

0.28

Tabla 2.-Distribución de los tipos cefálicos en los distintos sectores de la superticie del cuerpo. $\mathrm{k}$-número de zonas ocupadas por cada tipo: $\mathrm{j}$-número de tipos que aparecen en cada zona; $\mathrm{n}$-número de individuos. $\mathrm{H}$-índice de diversidad de Shannon-Weaver.

Distribution of anchor types among body surface sectors. $\mathrm{k}$ -number of anchor types found in each sector: $n$-number ot parasites. $\mathrm{H}$-Shannon-Weaver diversity index.

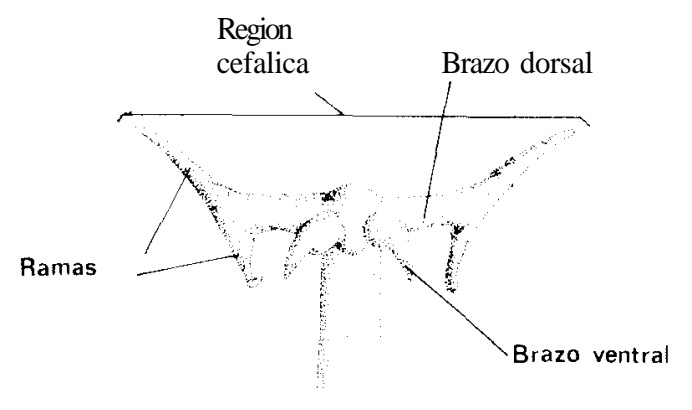

_. Abdomen

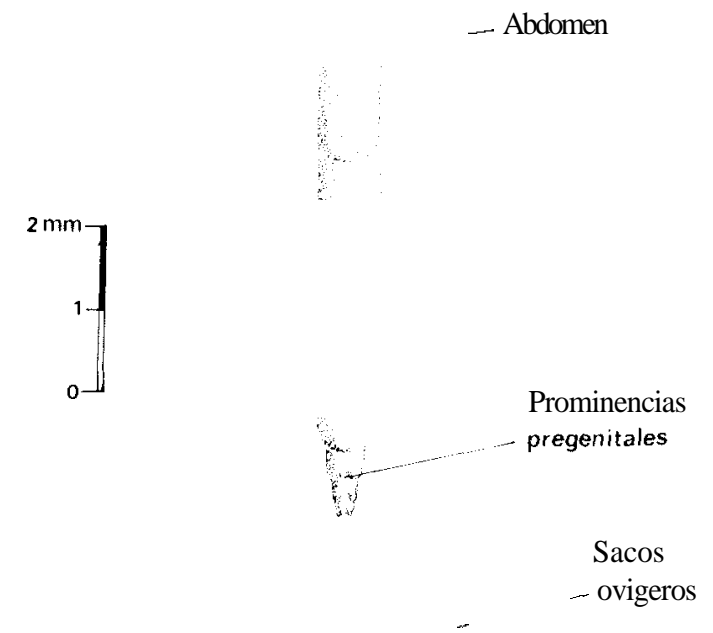

Figura 1.-Morfología externa de Lernaea cyprinacea L External morphology of Lernaea cyprinacea $L$.

gía de la región cefalica. El valor del test $\mathbf{G}$ de independencia fue de 9.8. no significativo ( $P<0.05)$, por lo que se pueden considerar independientes ambas variables. El número de prominencias pregenitales no es, por tanto. un carácter fijado a la morfología de la región cefálica y depende de las condiciones ambientales y de los hospedadores (Eisen. 1981: Tabla 1).

Para estudiar la relación entre la morfología y las características del lugar de inserción sobre el pez. se obtuvo el número de tipos cefálicos en cada zona del pez y se halló el índice de diversidad de ShannonWeaver de los tipos que aparecen en cada zona. De igual forma, se obtuvo el número de zonas ocupadas por cada tipo y el indice de diversidad de zonas por tipo (Tabla 3).

La diversidad de formas es mayor en las zonas 1.3. 8. 10. 31 y 26 , correspondiendo a las branquias. tres zonas del cuerpo y dos de las aletas y coinciden con 


\begin{tabular}{|c|c|c|c|c|c|c|c|c|c|}
\hline $\begin{array}{l}\text { Tipos } \\
\text { cefálicos }\end{array}$ & BRA & CUE & $\mathrm{AD}$ & $\mathrm{AL}$ & ALT & TOT & $X^{2}$ & $X^{2}$ & $\mathrm{H}$ \\
\hline 1 & 1 & 13 & 2 & 4 & 6 & 20 & $20^{\prime \prime \prime}$ & $05^{\prime \prime \prime}$ & 043 \\
\hline 2 & 0 & 8 & 4 & 3 & 6 & 14 & $223^{x}$ & $31 " '$ & 042 \\
\hline 3 & 0 & 21 & 1 & 0 & 1 & 22 & $70^{\mathrm{ns}}$ & $61^{x}$ & 008 \\
\hline 4 & 1 & 2 & 0 & 0 & 0 & 3 & & & \\
\hline 5 & 1 & 3 & 1 & 0 & 1 & 5 & $75^{\mathrm{ns}}$ & $33^{\text {nls }}$ & 041 \\
\hline 6 & 0 & 1 & 0 & 0 & 0 & 1 & & & \\
\hline 7 & 0 & 2 & 0 & 0 & 0 & 2 & & & \\
\hline 8 & 0 & 1 & 0 & 0 & 0 & 1 & & & \\
\hline 9 & 0 & 5 & 1 & 3 & -1 & 9 & $26^{\prime \prime \prime}$ & $23^{\mathrm{nb}}$ & 041 \\
\hline 10 & 0 & 3 & 5 & 4 & 9 & 12 & $478^{x}$ & $17.2^{x}$ & 047 \\
\hline 11 & 0 & 1 & 2 & 0 & 2 & 3 & & & \\
\hline 12 & 1 & 0 & 0 & 0 & 0 & 1 & & & \\
\hline 13 & 1 & 0 & 0 & 0 & 0 & 1 & & & \\
\hline
\end{tabular}

Tabla 3.-Frecuencia de aparición de los tipos cefálicos en branquias (BRA), superficie del cuerpo (CUE), aleta dorsal (AD), restode aletas (AL), y total de aletas (ALT). $\mathrm{X}^{2}{ }_{\mathrm{a}}$ - chi-cuadrado considerando AD y AL; $\mathrm{X}^{2}{ }_{\mathrm{t}}$ - chi-cuadrado considerando ALT.H-Indice de diversidad de Shannon-Weaver.

Frequency of appearance of anchor types in gills (BRA). body surface (CUE), dorsal fin (AD), other fins (AL) and fins, overall $(\mathrm{ALT}) ; \mathrm{X}^{2}{ }_{\mathrm{a}}$-value ofstatistic for the classes $\mathrm{AD}$ and $\mathrm{AL} ; \mathrm{X}^{2}{ }_{1}$ - for ALT; $\mathrm{H}$ - Shannoii-Weaver diversity index.
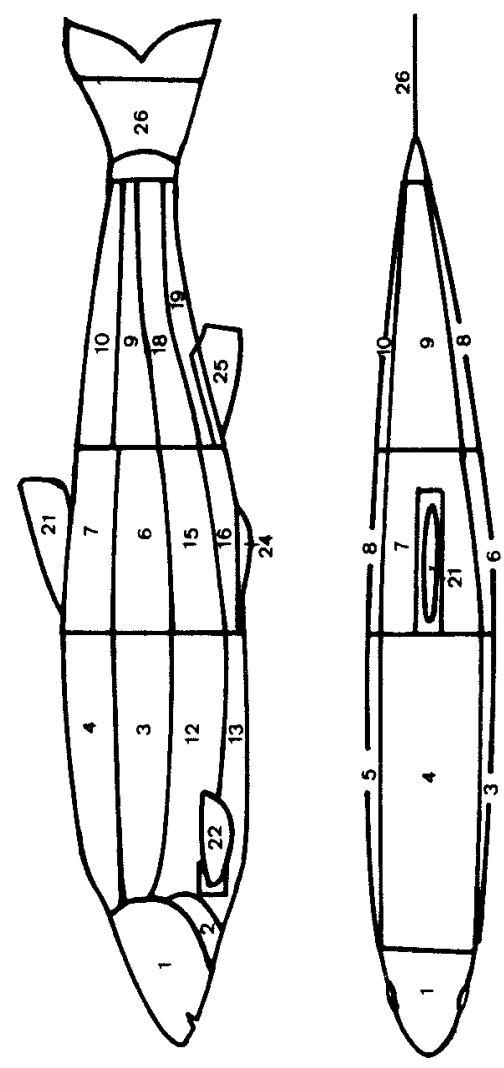

Figura 2.-Zonas de la superficie del pez utilizadas en el estudio. Sectors ofbody surface employed in this study.
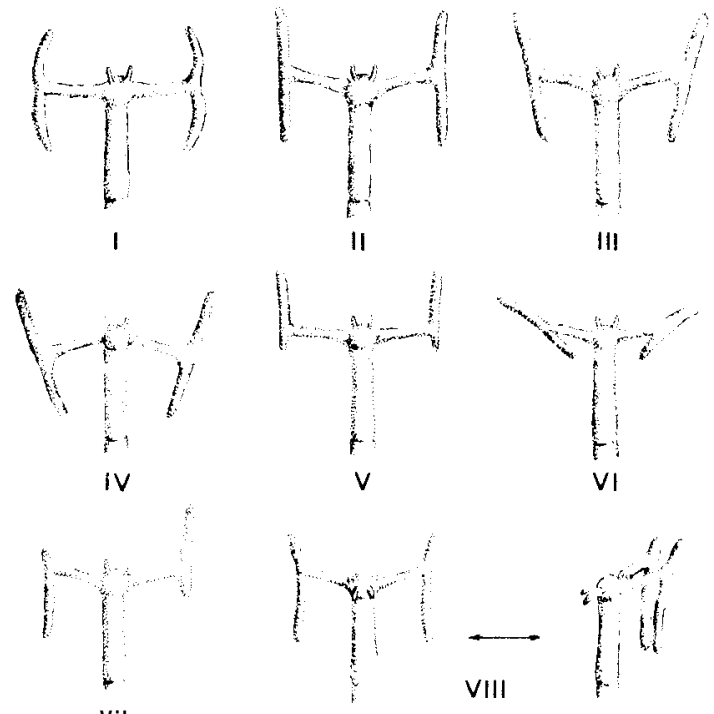

Vil

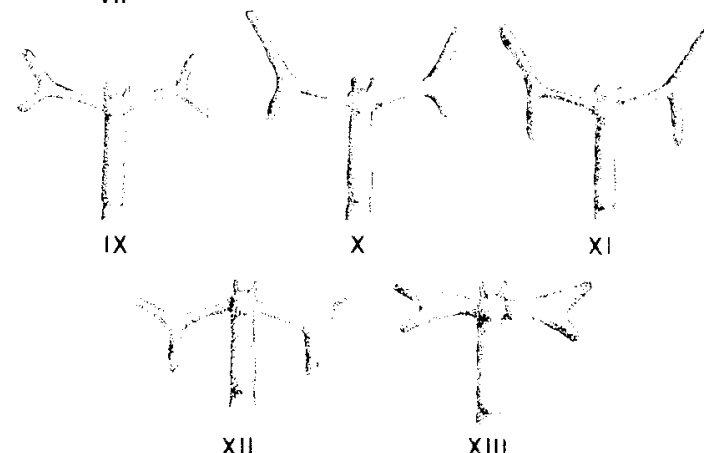

Figura 3.---Variaciones mortologicas de la región cefálica en Lemacaciprmacea.

Morphological variations of anchor in Lemaca ciymmated. 
las que presentan mayor número de individuos insertos. Con respecto a la diversidad de zonas en las que aparece cada tipo, toman valores más altos los tipos $1,2,3,5,9$ y 10 .

Los tres primeros corresponden a las formas más abundantes de L. cvprinacea, 5 y 10 son análogos, presentando reducción de la rama inferior de cada brazo y el 9 corresponde con la formatípica en $Y$.

Comparando las frecuencias de cada tipo aparecidas en las distintas zonas del pez, agrupadas en : branquias. superficie del cuerpo y aletas (distinguiendo dentro de éstas la aleta dorsal y el resto de las aletas) con las frecuencias de los distintos tipos esperados ell cada región, suponiendo una distribución homogénea. El mismo procedimiento se realizó para las frecuencias de zonas en las que aparece cada tipo. Las comparaciones se realizaron mediante test $\mathrm{X}^{2}$ (Tabla $3)$.

El tipo 3 aparece significativamente más abundante en el cuerpo que en las aletas, mientras que el tipo 10 ocurre más frecuentemente en las aletas que en el resto del cuerpo.

Las branquias no presentan diferencias con respecto a una distribución homogénea de tipos. lo que indica que permite variabilidad de la región cefálica en los parásitos insertos en ella, mientras que el resto de zonas del cuerpo y aletas presentan diferencias en los tipos. En el cuerpo aparecen por encima de la frecuencia media los tipos 1, 2, 3 y 9; en la aleta dorsal el 2 y 10 y en el resto de aletas el 1 y 10.

Los tipos 3 y 10 resultan, por tanto, específicos del cuerpo y las aletas respectivamente, mientras que los tipos 1, 2 y 9 se presentan con igual frecuencia en todas las zonas.

\section{DISCUSION}

Aún cuando Harding (op. cit.) incluye a L. elegans y L. cyprinacea en la misma especie, considera que existe una forma oriental en $Y$ y una forma occidental en $T$ dentro de ella. La plasticidad de Lernaea parece, sin embargo, ser mayor. Fryer (1961) considera que la variabilidad entre las distintas formas (o subespecies consideradas por $\mathrm{Hu}$ ) es mayor que la que presenta L. harnimiana en distintos sitios de un mismo hospedador. Esto está en contradicción con las observaciones de Poddubnaya (op. cit.) que considera la forma oriental como poco variable frente a la oriental.

En América, Amin (1981) apoya esta variabilidad. Este autor encontró individuos de L. cyprinacéa en los que aparecía. con un $70 \%$ la forma en T. un $15 \%$ la forma en $Y$ y un $15 \%$ un brazo en $T$ y el otro en $Y$. La proporción de formas $\boldsymbol{Y}$ y $T$ encontrada por nosotros varia con respecto a los resultados antes expues- tos, pero se observa mayor variabilidad dentro de un hospedador de la que se esperaría para las formas oriental yoccidental del parásito.

En relación con la especificidad por el hospedador, L. cyprinacea aparece en gran cantidad de familias (Haley \& Winn, 1959; Fratello et al. (op. cit.); Amin et al., 1973; etc.), presentándose en Arrocampo en varias especies de ciprinidos, en contradiccion con la especificidad observada por Poddubnaya (op. cit.).

Las relaciones entre el lugar de inserción del parásito y la morfología de la región cefálica, ha sido apuntada por Fryer (1961). Las observaciones de L. cyprinacea sobre boga corroboran estos resultados, apareciendo mayor diversidad de formas en la superficie del cuerpo. Las branquias presentan, sin embargo, un mayor número de formas posibles, mientras que en las aletas y superficies del cuerpo existe una forma dominante, la cual se presenta en otras zonas con frecuencias significativamente bajas. La forma de los parásitos insertos en la base de las aletas se caracteriza por un acortamiento de la rama inferior del brazo dorsal. Las que se presentan en la superficie del cuerpo no tienen, sin embargo, impedimento para el crecimiento en el plano de las escamas y permite mayor diversidad de formas (Fryer, 196 1).

Aunque pueda existir en L. cyprinacea una morfología típica de la región cefálica, pueden aparecer importantes variaciones debidas al lugar de inserción sobre el pez. La forma que más frecuentemente se presenta en una zona determinada es el resultado de las adaptaciones a posteriori de los brazos al espacio disponible, una vez insertos. Este caracter no permite, por tanto, distinguir especies próximas taxonómicamente ya que sufre importantes variaciones con el medio.

Estos resultados de especificidad, variabilidad de la morfología de la región cefálica e inconsistencia de las prominencias pregenitales como caracter, nos lleva a considerar a L. elegans como sinónimo de L. cyprinacea. siendo esta última la especie que se presenta en Arrocampo.

\section{BIBLIOGRAFIA}

Amin, O.M. (1973): On preliminary survey of vertebrate ectoparasites in southeastern Wisconsin. . Med. Ent., 10(1): 1 10-1 11 .

1981: On the crustacean ectoparasites of fishes from southeast Wisconsin. Trans. Am. Microsc. Soc.. 10012): $142-150$.

Amin, O.N., Balsano, J.S. \& Pfalzgraf, K.A. (1973): Lernaea cyprinacea Linn (Copepoda: Crustacea) from Root River. Wisconsin fishes. Am.Midl. Nat., 89/21:484-487.

Eisen, S. (1981): Characteristics of anchor parasite 
form fishes of North Pond, Kelley Island, Ohio. Ohio J. Sci., 81(4): 180-181.

Fratello, B., Sabatini, M.A. \& Stefanelli, A. (1973): Cariología e sistematica di Lernaea cyprinacea $L$. (Crustacea: Copepoda). Acad. Naz. dei Lincei. Voll LIII (I-2): 209-213.

Fryer, G. (1956): A report on the parasitic Copepoda and Branchiura of the fishes of Lake Nyasa. Proc. Zool. Soc. Lond. 127(3): 293-344.

(1961): Variations and systematic problcms in a group of iernaeid copepod. Crustaceana, 2: 275-285. (1982): Free living stages of freshwater parasitic Copepoda. Die Binnengewäser, Bd XXVI/2: 344-367.

Grabda, J. (1963): Life cycle and morphogenesis of Lernaea cyprinacea (L.) . Act. Parasit. Pol., II. 169-198.

Haley, A.J. \& Winn, H.E. (1959): Observations on a lernaean parasite of freshwater fishes. Trans. Am Fish. Soc., 88: 128-129.

Harding, J.P. (1950): On some species of Lrrnuru
(Crustacea: Copepoda: Parasite of freshwater fish). Bull. Br. Mus. (Nat. Hist) Zool.. 1: 1-27.

Hoffmann, G.L. (1976): Parasites of freshwater fishes. IV. Miscellaneous. The anchor parasite (L. elegans) and related species. Fish Disease leaflet (Fish and Wildlife Serv. Wash., D.C.), 46: 1-8.

Leigh, Sharpe, W.H. (1925): Lernaea (Lernaeocera) elegans sp. nov. a parasitic copepod of Anguilla japonica. Parasitology, 17: 245-251.

Linnaeus, C. (1758): Svstema Naturae Tomo 1: 655-656.

Poddubnaya, A.V. (1974): Parasite copepods of the genus Lernaea of Pond fishes (Tesis Abstr.). Ministertvo Rybnogo Khozyaistva RSFSR Gosudarstvenn yi Nauchno-Issledovatel skii Institut Ozernogo I.Re nogo Rybnogo Khozyaistva (GOSNIORKC), Leningrad. USSR. 19 pp.

Shield, R.J. (1978): Procedures for the laboratory rearing of Lernaea cyprinacea. Crustaceana 35 (3): $259-264$. 